\title{
Chronic Orofacial Pain and Systemic Disorders - A Prospective Study from Chennai, Tamil Nadu
}

\author{
Deepigaa Manivasagam ${ }^{1}$, Arvind Muthukrishnan² \\ 1,2 Department of Oral Medicine and Radiology, Saveetha Institute of Medical \\ and Technical Sciences (SIMATS), Kuthambakkam, Tamil Nadu, India.
}

\section{ABSTRACT}

\section{BACKGROUND}

Orofacial pain conditions occur due to complex pathophysiology and are often associated with psychosocial comorbidities. The purpose of the study was to assess the association between orofacial pain and systemic disorders.

\section{METHODS}

The study was conducted in a university dental hospital setting covering patients visiting for the management of various orofacial pain conditions from October 2020 to February 2021. A total of 50 patients clinically diagnosed with chronic orofacial pain ( $>3$ months) were involved in the study.

\section{RESULTS}

In this prospective study, 50 orofacial pain patients were involved in the study and consisted of burning mouth syndrome [BMS (12\%)], orofacial neuralgia (26\%), temporomandibular disorder [TMDS (54\%)] and atypical facial pain (8\%). Chronic orofacial pain patients were more common in 21 - 30 years (30\%) with a female predilection (56\%). The most common systemic disorders were gastritis (28\%) followed by depression (17\%), diabetes mellitus (13\%).

\section{CONCLUSIONS}

Chronic orofacial pain has a significant impact on quality of life and daily functioning. Chronic orofacial pain is associated with systemic diseases like gastritis, depression and diabetes mellitus. A biopsychosocial approach for the diagnosis and management may address the multifactorial aetiology of orofacial pain conditions whilst limiting the economic and health-related burden.
Corresponding Author: Dr. Arvind Muthukrishnan, Saveetha Institute of Medical and Technical sciences (SIMATS), Kuthambakkam, Tamil Nadu, India.

E-mail:arvindm@saveetha.com

DOI: $10.14260 / j e m d s / 2021 / 748$

How to Cite This Article: Manivasagam D, Muthukrishnan A. Chronic orofacial pain and systemic disorders - a prospective study from Chennai, Tamil Nadu. J Evolution Med Dent Sci 2021;10(43):3694-3700, DOI: 10.14260/jemds/2021/748

Submission 03-06-2021, Peer Review 07-08-2021, Acceptance 13-08-2021, Published 25-10-2021.

Copyright (c) 2021 Deepigaa Manivasagam et al. This is an open access article distributed under Creative Commons Attribution License [Attribution 4.0 International (CC BY 4.0)]

\section{KEY WORDS}

Orofacial Pain, Neuropathic Pain, TMD, Gastritis, Depression, Diabetes 


\section{BACKGROUND}

International Association for Study of Pain (IASP) has defined pain as a sensory unpleasant and emotional experience associated with actual or potential tissue damage or described in terms of such damage. Orofacial pain is referred to as pain associated with the head and neck region and is divided into physical (axis 1) and psychological (axis 2). ${ }^{1}$ Musculoskeletal disorder [Myofacial pain dysfunction syndrome ( MPDS)], Temporomandibular disorders, pulpal pain of somatic origin, neuropathic pain which includes trigeminal neuralgia, glossopharyngeal neuralgia, postherpetic neuralgia and neurovascular disorders / headaches (e.g. migraine and temporal arteritis) ${ }^{2}$ are all grouped under the physical conditions of orofacial pain.

Burning mouth syndrome is a condition that causes a burning sensation or discomfort affecting the mouth, which usually occurs in clinically healthy mucosa. ${ }^{3}$ There are two types of BMS - primary and secondary. When the organic cause for oral burning cannot be identified, it is termed as primary BMS whereas the presence of any local or systemic pathology gives rise to secondary BMS. ${ }^{3}$ Studies have stated that diabetic ${ }^{4}$ and hypertensive patients ${ }^{5}$ tend to acquire the condition more frequently. For accurate identification of the systemic causes of BMS, haematological and biochemical investigations should be done to rule out anaemia and the deficiency of other hematinics. ${ }^{6}$ Research shows that BMS has affected 0.7 - $15 \%$ of the population with a slightly high female predilection. ${ }^{7}$

The conditions that affect the temporomandibular joint (TMJ), and / or the masticatory muscles are referred to as temporomandibular joint disorders (TMD). ${ }^{8} \mathrm{~A}$ proper patient history and examination is essential to arrive at the diagnosis of TMD. History taking comprises information about the nature of pain, precipitating and aggravating factors, habits and any existing or prior trauma. ${ }^{9,10}$ Patient examination involves the clinical assessment of mouth opening, TMJ palpation and joint sound auscultation. Tenderness or hypertrophy is elicited by palpation of the muscles of mastication.

Pain that is caused by a lesion or disease of the somatosensory nervous system is described as Neuropathic orofacial pain neuralgia. Trigeminal Neuralgia (TN) is a neuropathic condition ${ }^{11}$ which is defined as constant unilateral facial pain that varies in intensity, aggravated by trigger factors. ${ }^{12}$ It occurs when one or more branches of the trigeminal nerve are affected. It is often characterized by episodic pain and is of short duration, with a sudden onset and decrease. The nature of the pain is usually excruciating which is often described by patients as stabbing pain like that of an electric shock, which occurs spontaneously, or when triggered by factors like touch, eating or wind. ${ }^{11} \mathrm{TN}$ is categorised into classical $\mathrm{TN}$ and symptomatic $\mathrm{TN}$ by the International Headache Society (IHS). ${ }^{13}$ Classical TN is due to neurovascular compression, commonly superior cerebellar pontine artery. Symptomatic TN is caused due to anatomical lesions excluding vascular compressions, like multiple sclerosis (MS) or space-occupying lesions. ${ }^{13}$ The current classification of TN suggests a third diagnostic category termed idiopathic TN (i.e.) there is no compression or pathology identified. ${ }^{14}$
Studies have stated the incidence rate of TN to be 27 per 100,000 . Glossopharyngeal neuralgia (GPN) is a condition characterised by recurring episodes of intense, jabbing pain experienced in the ear, the base of the tongue, the tonsillar area, and or beneath the angle of the jaw. Usually, they are unilateral, bilateral pain is experienced by $25 \%$ of patients. ${ }^{15}$ Incidence of GPN is estimated between 0.2 and 0.7 per 100,000 individuals per year. ${ }^{15}$ The regrowth of varicellazoster virus (V2V) within cranial sensory ganglia results in the Herpes zoster (HZ). $16-22 \%$ of patients with acute HZ will report pain for 3 to 6 months. It results in a direct complication of acute $\mathrm{HZ}$ of the trigeminal nerve and is restricted to the affected dermatome. ${ }^{16}$

Orofacial pain contemplated as a multidimensional phenomenon affects the quality of life and is always challenging for the clinician for diagnosing because of the lack of consensus regarding diagnostic criteria. Adoption of a biopsychosocial approach that helps in early and accurate diagnosis and a comprehensive approach to management is essential to limit the negative impact of orofacial pain psychosocially, whilst ensuring that the care remains effective and efficient.

The most common comorbidities reported with orofacial pain are psychiatric disorders and fibromyalgia and usually associated symptoms are gastrointestinal disorders and functional symptoms. ${ }^{17,18}$ Thus the objective of the study was to determine the association between orofacial pain and systemic disorders amongst patients visiting our university hospital.

\section{METHODS}

A prospective study was conducted in a university dental hospital setting covering patients visiting for the management of various orofacial pain conditions. The research was approved by the institutional ethical committee and scientific review board (SRB). The study was carried out by 2 members, the primary researcher and a department faculty. The study was carried out in the oral medicine department from October 2020 to February 2021. Sample size calculation was done using $\mathrm{G}$ power analysis software and calculated to the power of 80 . A total of 50 clinically diagnosed chronic orofacial pain ( $>3$ months) patients were involved in the study. The case sheets were cross verified based on clinical diagnosis, lab reports and radiographic findings. A complete medical history was obtained. The data was verified by both researchers. The internal validity maintained as set diagnostic criteria was followed and this methodology could be replicated at other centres also, maintaining the external validity as well.

\section{Statistical Analysis}

The data was formulated on an excel sheet then later transferred to an SPSS file. The statistical software used for analysis was IBM SPSS 23. The qualitative variables used in the study were gender, orofacial pain disorders like burning mouth syndrome (BMS), orofacial neuralgia, temporomandibular disorders (TMD), atypical facial pain and systemic disorders. Quantitative variables included the age. 
Descriptive analysis was performed for the independent and dependent variables as mentioned above. The type of analysis used was correlation and association and chi-square test was performed to evaluate the level of significance.

\section{RESULTS}

In this study, 50 orofacial pain patients were involved who consisted of BMS (12\%), orofacial neuralgia (26\%), TMDS (54 \%) and atypical facial pain (8\%) [Graph 1]. The frequency distribution of age and sex of chronic orofacial pain patients were 11 - 20 years (6\%), 21 - 30 years (30\%), 31 40 years $(16 \%), 41-50$ years $(16 \%), 51-60$ years $(16 \%)$, $61-70$ years (16\%) with a female predilection (56\%) and male (44\%). Chi-square test for age and chronic orofacial pain revealed TMD as more common in $21-30$ yrs., orofacial neuralgia in 51 to 60 years, BMS in 41 to 50 years and atypical facial pain in 61 to 70 years with a significant $P$-value
(Pearson chi-square $=0.000)$. $[$ Graph 2] and the association between sex and chronic orofacial pain revealed that TMD had a male predilection while orofacial neuralgia, BMS, atypical facial pain were more common in females; $\mathrm{P}<0.05$ ( Pearson chi square $=0.021)$. $[$ Graph -3$]$.

The most common systemic disorders reported in our study population were gastritis (28 \%) followed by depression (17\%), diabetes (13\%), hypertension (11\%) and heart diseases in $10 \%$ while the least reported were sinusitis (8\%) followed by asthma (6\%), rhinitis (4\%), thyroid (2\%) and nephrological disorders in $1 \%$ [Graph 4].

Association of systemic disorders with chronic orofacial pain revealed gastritis was more commonly associated with TMD, BMS and atypical facial pain i.e. $33 \%, 46 \%$ and $29 \%$ respectively followed by depression in $30 \%, 20 \%$ and $22 \%$ respectively. Diabetes (23\%), hypertension (20\%) and heart diseases $(20 \%)$ showed an increased association with orofacial neuralgias [Graph 5]

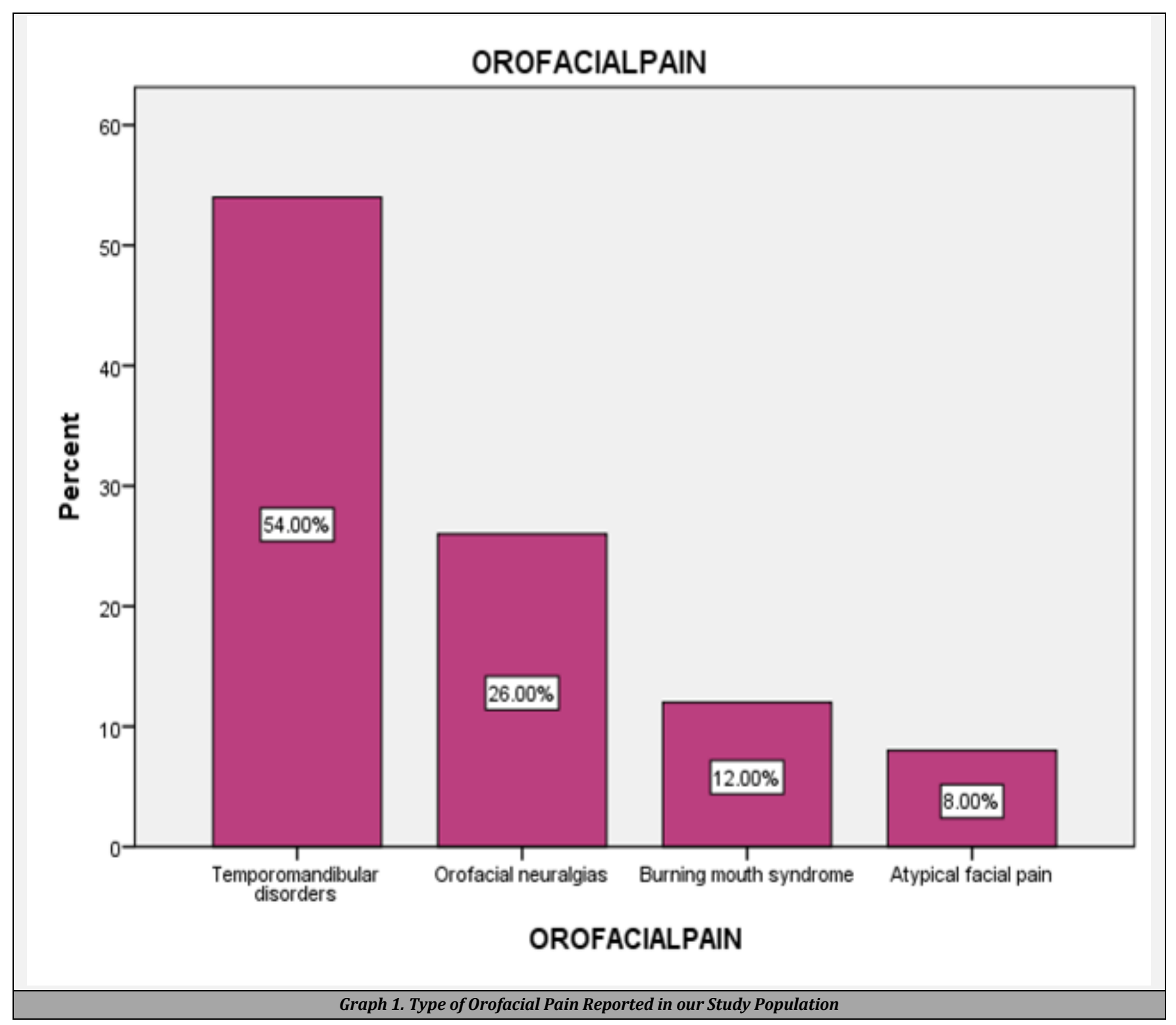



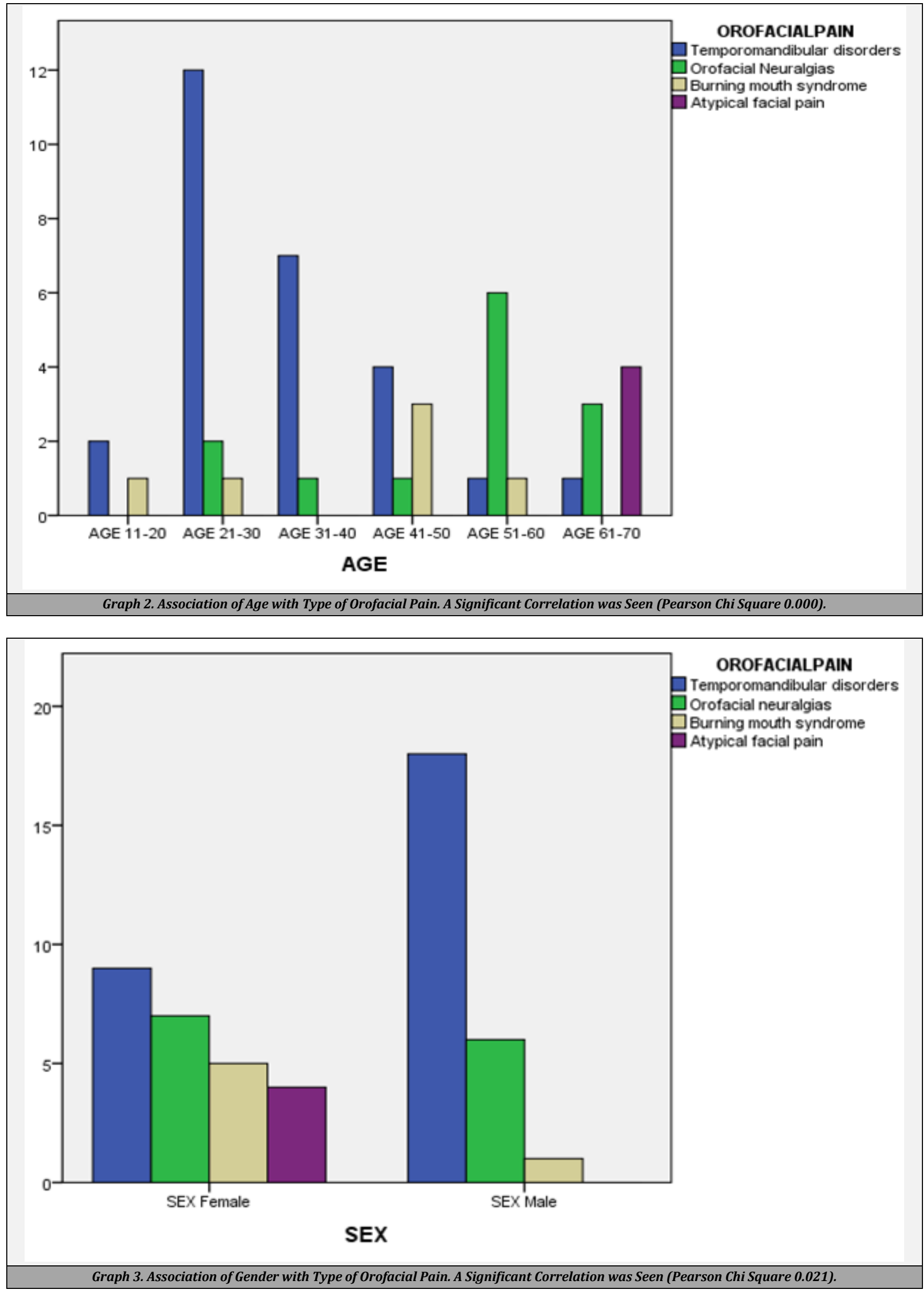


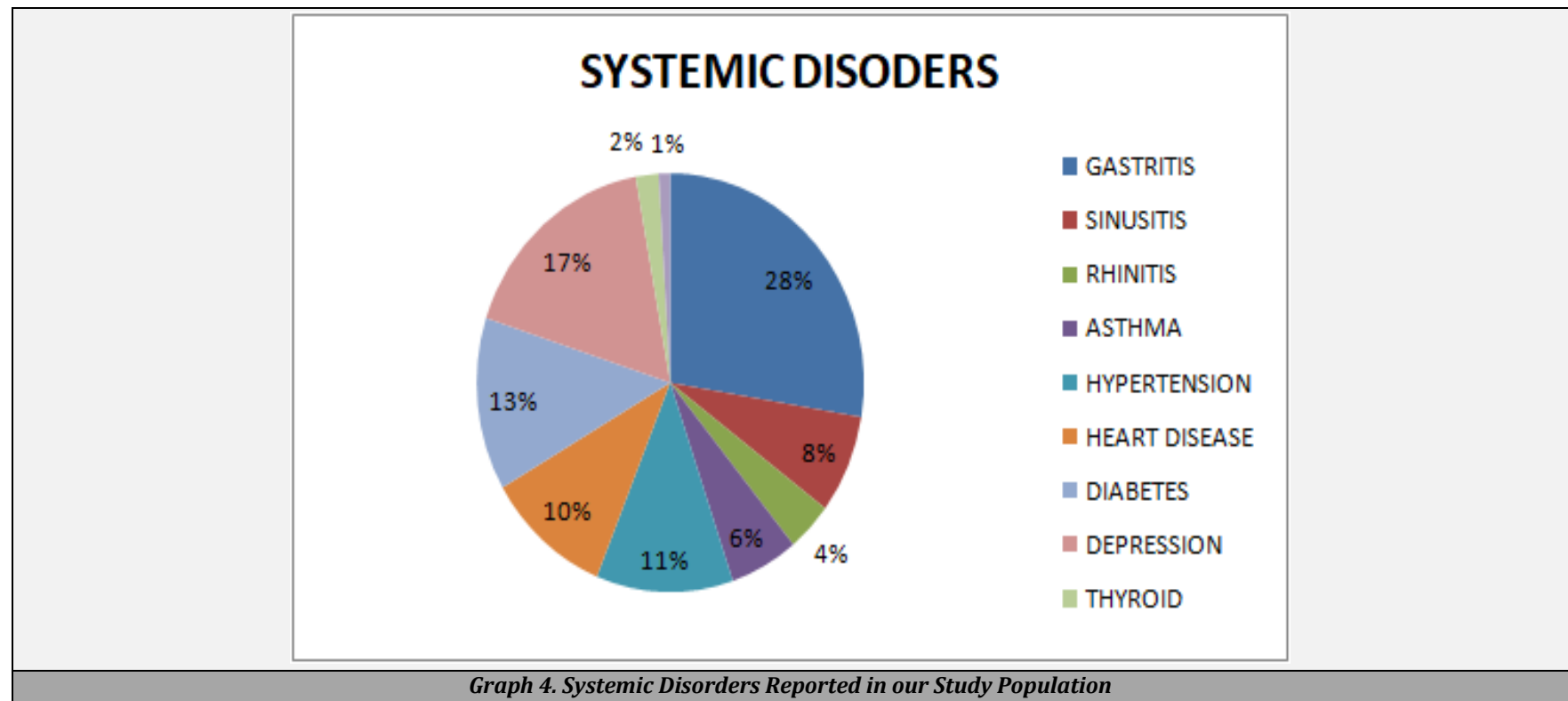

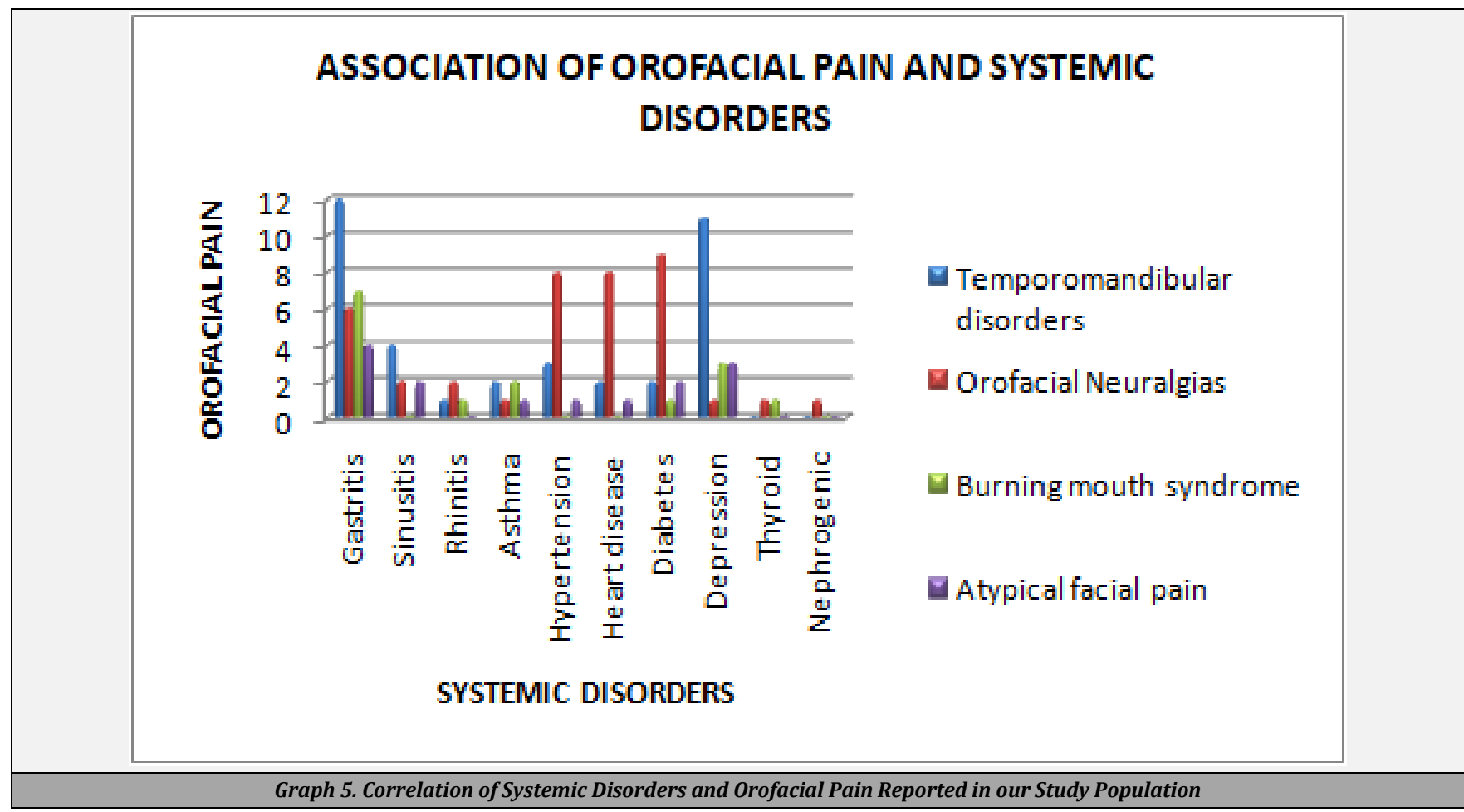

\section{DISCUSSION}

Orofacial pain is described as pain that arises from regions of the face and mouth and the most common causes include diseases of regional structures, disorders of the nervous system, referral pain from distant sources or sometimes of idiopathic aetiology. ${ }^{19}$ When pain is present for less than 3 months it is termed as acute and pain that persists for more than 3 months is termed as chronic. The diagnosis and management of orofacial pain are difficult due to intricate patient histories, pathophysiology, psychosocial comorbidities and associated underlying systemic disorders.

The most common orofacial pain reported in our study was TMD which was similar to a study conducted by Derafshi
R et al. and Reyes et al. ${ }^{20,21}$ According to a study conducted by Macfarlane et al. orofacial pain was more prevalent among 18 to 25 years and less common in patients above 60 years which was similar to our study population. ${ }^{22}$ Orofacial pain was more prevalent among women in our study. According to a study conducted by Fillingim et al. women are more sensitive when compared to men and that may serve as an increased risk factor and they also have a lower pain threshold as reported by Riley and colleagues.23,24 Gauer and Semidey reported that TMD was more common in 20 to 40 years, while Tomattis et al. reported BMS was commonly seen in 41 to 50 years and these results were similar to our study. 25,26

Neuropathic pain was more commonly observed in 51 60 years. Korczeniewska O.A et al. in 2018 have reported more prevalence in 60 - 69 years. ${ }^{27}$ A significant correlation was observed between age, gender and type of orofacial pain. 
In our study, gastritis and depression were more commonly associated with TMD, BMS and atypical facial pain while depression and heart diseases were frequently reported with orofacial neuralgias. Literature has reported that gastritis and psychiatric disorders were more prevalent in TMD patients while nonfunctional illness showed increased association with neuropathic facial pain. ${ }^{28-31}$ Mariana et al. reported that gastritis and hypothyroidism were predictors of nonneuropathic orofacial conditions.

\section{CONCLUSIONS}

OFP constitutes a substantial spectrum of disorders due to their distinctive anatomical, physiological, and biochemical components. In most of the patients, it is not an isolated entity and is often accompanied by systemic comorbidities. Depression was reported in $17 \%$ of the study population and the new interesting finding was the presence of gastritis and diabetes at significant levels in patients with orofacial pain. Diagnosis and management of orofacial pain demand a biopsychosocial approach, which explains the multifactorial aetiology of the condition and thereby limits the associated health-related and economic burden. Thus, further research and follow up need to be done to evaluate if relief from orofacial pain is achieved if accompanying systemic disorders are treated.

Data sharing statement provided by the authors is available with the full text of this article at jemds.com.

Financial or other competing interests: None.

Disclosure forms provided by the authors are available with the full text of this article at jemds.com.

The authors are forever indebted to Saveetha Institute of Medical and Technical Sciences as permission was granted to conduct the present study and also to the faculty of the Department of Oral Medicine for their expert opinion, guidance and support rendered in the development and writing of these manuscripts.

\section{REFERENCES}

[1] Okeson JP. The classification of orofacial pains. Oral Maxillofac Surg Clin North Am 2008;20(2):133-44, v.

[2] Steele JC, Clark HJ, Hong CHL, et al. World Workshop on Oral Medicine VI: an International validation study of clinical competencies for advanced training in oral medicine. Oral Surg Oral Med Oral Pathol Oral Radiol 2015;120(2):143-51.e7.

[3] McMillan R, Forssell H, Buchanan JA, et al. Interventions for treating burning mouth syndrome. Cochrane Database Syst Rev 2016;11(11):CD002779.

[4] Coculescu EC, Părlătescu I, Gheorghe C, et al. Management of burning mouth syndrome (BMS) in patients with diabetes mellitus. Romanian J Military Medicine 2017;119:43-51.

[5] de Sousa LMCM, de Morais NFLDAC, Neto JDC, et al. Burning mouth syndrome secondary to antihypertensive drug use: clinical case report. Oral Surg Oral Med Oral Pathol Oral Radiol 2015;120(2):e58-9.

[6] Renton T, Durham J, Aggarwal VR. The classification and differential diagnosis of orofacial pain. Expert Rev
Neurother 2012;12(5):569-76.

[7] Patton LL, Siegel MA, Benoliel R, et al. Management of burning mouth syndrome: systematic review and management recommendations. Oral Surg Oral Med Oral Pathol Oral Radiol Endod 2007;(Suppl 103):S39.e1-13.

[8] Durham J, Wassell RW. Recent advancements in Temporomandibular Disorders (TMDs). Rev Pain 2011;5(1):18-25.

[9] Durham J. Summary of Royal College of Surgeons' (England) clinical guidelines on management of temporomandibular disorders in primary care. Br Dent J 2015;218(6):355-6.

[10] Choudhury P, Panigrahi RG, Maragathavalli G, et al. Vanishing roots: first case report of idiopathic multiple cervico - apical external root resorption. J Clin Diagn Res 2015;9(3):ZD17-9.

[11] Zakrzewska JM, Linskey ME. Trigeminal neuralgia. $\mathrm{Br}$ Med J 2014;348:g474.

[12] Subha M, Arvind M. Role of magnetic resonance imaging in evaluation of trigeminal neuralgia with its anatomical correlation. Biomedical and Pharmacology Journal 2019;11(1):289-96.

[13] Olesen J, Steiner TJ. The International classification of headache disorders, 2nd edn (ICDH - II). J Neurol Neurosurg Psychiatry 2004;75(6):808-11.

[14] Cruccu G, Finnerup NB, Jensen TS, et al. Trigeminal neuralgia: new classification and diagnostic grading for practice and research. Neurology 2016;87(2):220-8.

[15] Katusic S, Williams DB, Beard CM, et al. Incidence and clinical features of glossopharyngeal neuralgia, Rochester, Minnesota, 1945-1984. Neuroepidemiology 1991;10(5-6):266-75.

[16] Christoforou J. Neuropathic orofacial pain. Dent Clin North Am 2018;62(4):565-84.

[17] Fadgyas-Stanculete M, Ana-Maria B, Popa-Wagner A, et al. The relationship between irritable bowel syndrome and psychiatric disorders: from molecular changes to clinical manifestations. J Mol Psychiatry 2014;2(1):4.

[18] Turk DC, Fillingim RB, Ohrbach R, et al. Assessment of psychosocial and functional impact of chronic pain. J Pain 2016;17(Suppl 9):T21-49.

[19] Benoliel R, Svensson P, Evers S, et al. The IASP classification of chronic pain for ICD - 11: chronic secondary headache or orofacial pain. Pain 2019;160(1):60-8.

[20] Derafshi R, Rezazadeh F, Ghapanchi J, et al. Prevalence of chronic orofacial pain in elderly patients referred to shiraz dental school from 2005 to 2017. Anesth Pain Med 2019;9(6):e91182.

[21] Romero-Reyes M, Uyanik JM. Orofacial pain management: current perspectives. J Pain Res 2014;7:99-115.

[22] Macfarlane TV, Blinkhorn AS, Davies RM, et al. Orofacial pain in the community: prevalence and associated impact. Community Dent Oral Epidemiol 2002;30(1):5260.

[23] Fillingim RB, Maixner W. Gender differences in the responses to noxious stimuli. Pain Forum 1995;4(4):209-21.

[24] Riley JL 3rd, Robinson ME, Wise EA, et al. Sex differences in the perception of noxious experimental stimuli: a meta - analysis. Pain 1998;74(2-3):181-7. 
[25] Gauer RL, Semidey MJ. Diagnosis and treatment of temporomandibular disorders. Am Fam Physician 2015;91(6):378-86.

[26] de Serrano OSTP, Munerato MC. Burning mouth syndrome- latest update. International Journal of Dentistry Research 2016;1(1):14-23.

[27] Korczeniewska OA, Eliav E, Benoliel R. Neuropathic orofacial pain. In: Farah CS, Balasubramaniam R, McCullough MJ, eds. Contemporary oral medicine: a comprehensive approach to clinical practice. New York, NY: Springer International Publishing 2018: p. 1-75.

[28] Klaus K, Fischer S, Doerr JM, et al. Classifying fibromyalgia syndrome as a mental disorder? An ambulatory assessment study. Int J Behav Med 2017;24(2):230-8.

[29] Bazzichi L, Giacomelli C, Consensi A, et al. One year in review 2016: fibromyalgia. Clin Exp Rheumatol 2016;34(2 Suppl 96):S145-9.

[30] Hungin APS, Becher A, Cayley B, et al. Irritable bowel syndrome: an integrated explanatory model for clinical practice. Neurogastroenterol Motil 2015;27(6):750-63.

[31] Abrahamsen R, Baad-Hansen L, Svensson P. Hypnosis in the management of persistent idiopathic orofacial painclinical and psychosocial findings. Pain 2008;136(12):44-52. 